\title{
THE PERIODIC BEHAVIOR OF MORSE-SMALE DIFFEOMORPHISMS ON COMPACT SURFACES
}

BY

\author{
CAROLYN C. NARASIMHAN
}

\begin{abstract}
Necessary and sufficient conditions are given for the existence of Morse-Smale diffeomorphisms homotopic to the identity with prescribed periodic characteristics on any compact 2-manifold.
\end{abstract}

An important class of dynamical systems on smooth compact manifolds consists of the Morse-Smale diffeomorphisms. These have a relativety simple orbit structure and this structure is preserved under small $C^{1}$ perturbations. If $f$ is a Morse-Smale diffeomorphism, then the nonwandering set of $f$ consists of a finite number of periodic orbits $\gamma_{i}$ and each such $\gamma_{i}$ has the following invariants: $p_{i}$, the period of $x \in \gamma_{i}$; $u_{i}$, the dimension of $E_{x}^{u_{i}}$ for $x \in \gamma_{i}$; and $\Delta_{i}$, the orientation type of $x \in \gamma_{i}$ (see $\$ 1$ for definitions). If we define the periodic data of a diffeomorphism to be the collection of triples $\left(p_{i}, u_{i}, \Delta_{i}\right)$, then it would be interesting to know when there exists a diffeomorphism with given periodic data.

Clearly, the topology of a manifold $M$ imposes certain restrictions on the orbit structure of a diffeomorphism of $M$. For example, if $M$ is compact, then a Morse-Smale diffeomorphism of $M$ has at least one source and one sink; that is, $u_{i}=0$ and $u_{j}=\operatorname{dim} M$ for some $i$ and $j$.

Smale defines in [9] the homology zeta function $\eta(f)$ of a diffeomorphism $f$ of a compact manifold $M$ by

$$
\eta(t)=\exp \left(\sum_{m=1}^{\infty} \frac{1}{m} L\left(f^{m}\right) t^{m}\right)
$$

where $L\left(f^{m}\right)$, the Lefschetz number of $f^{m}$, counts the fixed points of $f^{m}$ algebraically. This function is always rational and is a homotopy invariant of $f$. In fact, as shown in [9], if $f$ is homotopic to the identity of $M$, then $\eta(f)=(1-t)^{-\chi(M)}$, where $\chi(M)$ is the Euler characteristic of $M$. Franks has shown in [3] that if $f$ is a $C^{1}$ map with only $n$ periodic orbits, all hyperbolic,

Received by the editors July 19, 1977 and, in revised form, November 22, 1977.

AMS (MOS) subject classifications (1970). Primary 58F20; Secondary 55C20. 
then $\eta(f)$ has the simple form

$$
\eta(t)=\prod_{i=1}^{n}\left(1-\Delta_{i} t^{p_{i}}\right)^{(-1)^{u_{i}+1}}
$$

where $\left\{\left(p_{i}, u_{i}, \Delta_{i}\right)\right\}_{i=1}^{n}$ is the periodic data of $f$. Therefore, the periodic data of a Morse-Smale diffeomorphism $f$ which is homotopic to the identity must satisfy the equation

$$
\prod_{i=1}^{n}\left(1-\Delta_{i} t^{p_{i}}\right)^{(-1)^{u_{i}}}=(1-t)^{x(M)}
$$

The purpose of this paper is to show that when $M$ is a compact, connected 2-manifold, these two conditions are sufficient for the existence of a MorseSmale diffeomorphism homotopic to the identity. Thus, we have the following theorem.

THEOREM. Let $M$ be a compact, connected 2-manifold. There exists a Morse-Smale diffeomorphism $f$ of $M$ homotopic to the identity with periodic data $\left\{\left(p_{i}, u_{i}, \Delta_{i}\right)\right\}_{i=1}^{n}$ if and only if

(a) $u_{i}=0$ and $u_{j}=2$ for some $i$ and $j$;

(b) $\prod_{i=1}^{n}\left(1-\Delta_{i} t^{p_{i}}\right)^{(-1)^{u_{i}}}=(1-t)^{x(M)}$.

In $\S 1$, we give definitions and background. Two general references for this are [9] and [5]. In $\$ 2$, we consider the equation

$$
\prod_{i=1}^{n}\left(1-\Delta_{i} t^{p_{i}}\right)^{(-1)^{u_{i}}}=(1-t)^{x(M)}
$$

and, using combinatorial arguments, rewrite it in a canonical form which is useful in proving the theorem. In $\$ 3$, we construct several basic diffeomorphisms on discs and annuli in the plane and indicate how these can be pieced together to produce the desired diffeomorphism on the given 2-manifold. Some additional results derived from the canonical form are proved in $\$ 4$.

I wish to thank my advisor, John Franks, for suggesting the problem and for the help he has given me with this work, and R. Narasimhan, for helpful conversations.

1. Preliminaries. Let $M$ be a compact $C^{\infty}$ manifold and $\operatorname{Diff}(M)$ the group of $C^{r}$ diffeomorphisms of $M, 1 \leqslant r \leqslant \infty$. A point $x \in M$ is called a nonwandering point of $f \in \operatorname{Diff}(M)$ if for every neighborhood $U$ of $x$, there exists $m>0$ such that $f^{m}(U) \cap U \neq \varnothing$. The set of nonwandering points is a closed, invariant set of $f$ and is denoted by $\Omega(f)$, or $\Omega$.

A hyperbolic periodic point of $f$ is a point $x \in M$ with $f^{n}(x)=x$ for some $n \geqslant 1$ and such that the derivative map $D f_{x}^{n}: T_{x} M \rightarrow T_{x} M$ has no eigenvalues of absolute value one. If all eigenvalues of $D f_{x}^{n}$ are less than one in absolute 
value, $x$ is called a sink, and if all eigenvalues of $D f_{x}^{n}$ are greater than one in absolute value, $x$ is called a source. Otherwise, $x$ is called a saddle. Let $E_{x}^{u}$ be the subspace of $T_{x} M$ spanned by eigenspaces of $D f_{x}^{n}$ corresponding to eigenvalues greater than one in absolute value, and $E_{x}^{s}$ the subspace of $T_{x} M$ spanned by the remaining eigenspaces. Then $T_{x} M=E_{x}^{u} \oplus E_{x}^{s}$, and this splitting is invariant under $D f_{x}^{n}$.

Suppose $\gamma$ is a hyperbolic periodic orbit of $f$ with least period $p$. Let $x$, $y \in \gamma, f^{m}(x)=y$. Then $D f_{x}^{m}: E_{x}^{u} \rightarrow E_{y}^{u}$ is an isomorphism, so $\operatorname{dim} E_{x}^{u}=$ $\operatorname{dim} E_{y}^{u}$. Also, $D f_{x}^{p}: E_{x}^{u} \rightarrow E_{x}^{u}$ preserves orientation if and only if $D f_{y}^{p}: E_{y}^{u} \rightarrow$ $E_{y}^{u}$ preserves orientation, since

$$
D f_{y}^{p}=\left(D f_{x}^{m}\right)\left(D f_{x}^{p}\right)\left(D f_{x}^{m}\right)^{-1}
$$

Define the orientation type $\Delta$ of $\gamma$ to be +1 if $D f_{x}^{p}: E_{x}^{u} \rightarrow E_{x}^{u}$ preserves orientation and -1 if it reverses orientation.

Thus, each hyperbolic periodic orbit $\gamma_{i}$ of $f$ has three integer invariants: $p_{i}$, the least period of $\gamma_{i} ; u_{i}=\operatorname{dim} E_{x}^{u_{i}}$ for $x \in \gamma_{i}$; and $\Delta_{i}$, the orientation type of $x \in \gamma_{i}$.

Definition. If $f$ is a diffeomorphism whose nonwandering set consists of $n$ hyperbolic periodic orbits, we define the periodic data of $f$ to be the collection $\left\{\left(p_{i}, u_{i}, \Delta_{i}\right)\right\}_{i=1}^{n}$.

The stable manifold of a hyperbolic fixed point $x$ of $f$ is defined by

$$
W^{s}(x)=W^{s}(x, f)=\left\{y \in M \mid d\left(f^{n}(x), f^{n}(y)\right) \rightarrow 0 \text { as } n \rightarrow \infty\right\},
$$

where $d$ is a metric on $M$, and the unstable manifold of $x$ is $W^{u}(x)=$ $W^{u}(x, f)=W^{s}\left(x, f^{-1}\right)$. These are one-to-one immersed submanifolds of $M$ and $T_{x}\left(W^{s}(x)\right)=E_{x}^{s}, T_{x}\left(W^{u}(x)\right)=E_{x}^{u}$ (see [8]). If $x$ is a hyperbolic periodic point of period $p$, the stable and unstable manifolds of $x$ are defined by

$$
W^{s}(x)=W^{s}\left(x, f^{p}\right), \quad W^{u}(x)=W^{u}\left(x, f^{p}\right) .
$$

Definition. A Morse-Smale diffeomorphism is a diffeomorphism $f$ of $M$ satisfying the following conditions:

(1) $\Omega(f)$ is finite;

(2) the periodic points of $f$ are hyperbolic;

(3) for each $x, y \in \Omega(f), W^{s}(x)$ and $W^{u}(y)$ have transversal intersection. Condition (1) implies that $\Omega(f)$ consists of periodic points. Palis and Smale proved in [6] that for $f \in \operatorname{Diff}(M)$ with $\Omega(f)$ finite, $f$ is a Morse-Smale diffeomorphism if and only if $f$ is structurally stable.

For a Morse-Smale diffeomorphism, $M=\cup W^{s}\left(x_{i}\right)=\cup W^{u}\left(x_{i}\right)$ for $x_{i} \in$ $\Omega(f)$. Therefore, a Morse-Smale diffeomorphism has at least one source and one sink.

If $f \in \operatorname{Diff}(M)$ has a finite nonwandering set consisting of periodic orbits $\left\{\gamma_{i}\right\}_{i=1}^{k}$, then a filtration for $f$ is a finite sequence $\left\{\boldsymbol{M}_{i}\right\}_{i=1}^{k}$ of manifolds with 
boundary, of the same dimension as $M$, such that $M=M_{k} \supset \cdots \supset M_{1} \supset$ $M_{0}=\varnothing, f\left(M_{i}\right) \subset \operatorname{int}\left(M_{i}\right)$, and $\bigcap_{m \in \mathbf{Z}} f^{m}\left(M_{i}-M_{i-1}\right)=\gamma_{i}$.

In proving the theorem, we shall construct a diffeomorphism $f$ of $M$ whose nonwandering set is a finite number of hyperbolic periodic orbits and such that $f$ has a filtration. In this case, by the $\Omega$-stability theorem (see [10]), and the Kupka-Smale theorem (see [1] or [7]), we can find in a neighborhood of $f$ a Morse-Smale diffeomorphism of $M$ which has the same periodic data as $f$.

2. Canonical form. To prove that a diffeomorphism $f$ whose periodic data satisfy the equation

$$
\prod_{i=1}^{n}\left(1-\Delta_{i} t^{p_{i}}\right)^{(-1)^{u_{i+1}}}=\eta(f)=(1-t)^{-\chi(M)}
$$

can actually be constructed, it is necessary to study this equation more closely. First, note that in the case of a 2-dimensional manifold $M, u_{i}$ is 0,1 , or 2. Furthermore, since $f$ is homotopic to the identity, it is orientation preserving. Thus, $\Delta_{i}=+1$ when $u_{i}=0$ or $u_{i}=2$, and $\Delta_{i}$ may be +1 or -1 when $u_{i}=1$. Therefore, we are interested in equations of the form

$$
\frac{\Pi_{i=1}^{m}\left(1 \pm t^{q_{i}}\right)}{\prod_{j=1}^{l}\left(1-t^{p_{j}}\right)}=1 .
$$

We shall show that such an equation imposes restrictions on the integers $m, l$, $q_{i}$, and $p_{j}$ (which are part of the given periodic data).

LEMMA 2.1. If

$$
\frac{\Pi_{i=1}^{m}\left(1-t^{q_{i}}\right) \Pi_{k=1}^{n}\left(1+t^{r_{k}}\right)}{\Pi_{j=1}^{l}\left(1-t^{p_{j}}\right)}=1,
$$

then

(a) $m=l$;

(b) there exist integers $a_{1} \geqslant 0, \ldots, a_{m} \geqslant 0$ such that

(i) the set $\left\{p_{1}, p_{2}, \ldots, p_{m}\right\}$ equals the set $\left\{2^{a_{1}} q_{1}, 2^{a_{2}} q_{2}, \ldots, 2^{a_{m}} q_{m}\right\}$;

(ii) the set $\left\{r_{1}, r_{2}, \ldots, r_{n}\right\}$ equals the set

$$
\left\{q_{1}, 2 q_{1}, \ldots, 2^{a_{1}-1} q_{1} ; q_{2}, 2 q_{2}, \ldots, 2^{a_{2}-1} q_{2} ; \cdots ; q_{m}, \ldots, 2^{a_{m}-1} q_{m}\right\} .
$$

(If $a_{i}=0$ for some $i$, i.e., if $p_{i}=q_{i}$, then the set of numbers $q_{i}, \ldots, 2^{a_{i}-1} q_{i}$ in

(ii) stands for the empty set, i.e., there are no corresponding $r_{j}$.)

Thus, such an equation has the canonical form

$$
\prod_{i=1}^{m}\left[\frac{\left(1-t^{q_{i}}\right)\left(1+t^{q_{i}}\right)\left(1+t^{2 q_{i}}\right) \cdots\left(1+t^{2^{a_{i-1}}} \cdot q_{i}\right)}{\left(1-t^{2^{a} q_{i}}\right)}\right]=1 .
$$


Proof. Let $P(t)=\Pi_{i=1}^{m}\left(1-t^{q_{i}}\right) \Pi_{k=1}^{n}\left(1+t^{r_{k}}\right)$ and $Q(t)=\Pi_{j=1}^{l}\left(1-t^{p_{j}}\right)$. Then $P(t)$ has a zero of order $m$ at $t=1$ and $Q(t)$ has a zero of order $l$ at $t=1$. Since $P(t)=Q(t), m=l$.

We shall prove (b) by induction on $m+n$. Suppose $m+n=1$. Then either $m=0$ or $n=0$. In the first case, $\Pi_{k=1}^{n}\left(1+t^{r_{k}}\right)=1$, so $n=0$. In the second case, we have $\left(1-t^{q_{1}}\right)=\left(1-t^{p_{1}}\right)$. Therefore, $q_{1}=p_{1}$ and the statement is true in this case.

Now assume that the statement is true for $s<m+n$, and that we have

$$
\prod_{i=1}^{m}\left(1-t^{q_{i}}\right) \prod_{k=1}^{n}\left(1+t^{r_{k}}\right)=\prod_{j=1}^{m}\left(1-t^{p_{j}}\right) \text {. }
$$

Reorder the $q$ 's and $p$ 's so that

$$
q_{1} \leqslant q_{2} \leqslant \cdots \leqslant q_{m} \text { and } p_{1} \leqslant p_{2} \leqslant \cdots \leqslant p_{m} \text {. }
$$

We shall show that either $p_{m}=q_{m}$ or $p_{m}=2 r_{j}$ for some $j$.

Let

$$
P_{1}(t)=\prod_{i=1}^{m}\left(1-t^{q_{i}}\right), \quad P_{2}(t)=\prod_{k=1}^{n}\left(1+t^{r_{k}}\right) .
$$

Then $P_{1}(t) P_{2}(t)=Q(t)$. Since $e^{2 \pi i / q_{m}}$ is a root of $P_{1}(t)$, it is a root of $Q(t)$. Thus, there exists $j$ such that $e^{2 \pi i p_{j} / q_{m}}=1$. Therefore, $q_{m}$ divides $p_{j}$, so $q_{m} \leqslant p_{j} \leqslant p_{m}$. Now $e^{2 \pi i / p_{m}}$ is a root of $Q(t)$, so there are two possibilities to consider:

(i) Suppose $e^{2 \pi i / p_{m}}$ is a root of $P_{1}(t)$. Then, by the same reasoning as above, $p_{m} \leqslant q_{m}$, so $p_{m}=q_{m}=2^{0} q_{m}$. In this case, we have

$$
\prod_{i=1}^{m-1}\left(1-t^{q_{i}}\right) \prod_{k=1}^{n}\left(1+t^{r_{k}}\right)=\prod_{j=1}^{m-1}\left(1-t^{p_{j}}\right)
$$

and the result follows by induction,

(ii) Suppose $e^{2 \pi i / p_{m}}$ is a root of $P_{2}(t)$. Then there exists $j$ such that $e^{2 \pi i r_{j} / p_{m}}=-1$, so $2 r_{j} / p_{m}=l$ for some odd integer $l$. On the other hand, $e^{\pi i / r_{j}}$ is a root of $P_{2}(t)$, so it is a root of $Q(t)$ and $e^{\pi i p_{s} / r_{j}}=1$ for some $s$. Therefore, $p_{s} / r_{j}=2 l^{\prime}$ for some integer $l^{\prime}$. Thus, $p_{s}=l l^{\prime} p_{m}$. But $p_{s} \leqslant p_{m}$, so $l=1$ and $p_{m}=2 r_{j}$. In this case, we have

$$
\prod_{i=1}^{m}\left(1-t^{q_{i}}\right) \prod_{k=1}^{j-1}\left(1+t^{r_{k}}\right) \prod_{k=j+1}^{n}\left(1+t^{r_{k}}\right)=\left(1-t^{r_{j}}\right) \prod_{j=1}^{m-1}\left(1-t^{p_{j}}\right),
$$

or, after a change of notation,

$$
\prod_{i=1}^{m}\left(1-t^{q_{i}}\right) \prod_{k=1}^{n-1}\left(1+t^{r_{k}}\right)=\prod_{j=1}^{m}\left(1-t^{p_{j}}\right)
$$

and, again, the result follows by induction. Q.E.D. 
3. Construction of diffeomorphisms. The technique used in proving the sufficiency of the conditions in the theorem is to write the given 2-manifold $M$ as a union of 2-dimensional annuli, or annuli and discs, and to construct a diffeomorphism of $M$ which agrees with certain basic diffeomorphisms in these regions. Below, we prove the existence of three mappings of regions in the plane which, together with their inverses, will constitute these basic diffeomorphisms. These mappings are constructed so that they agree in a neighborhood of the boundaries of their domains, and therefore may be easily pieced together to define a mapping of the manifold $M$.

In the rest of this chapter, when we say that a diffeomorphism $f$ has periodic data $\left\{\left(p_{i}, u_{i}, \Delta_{i}\right)\right\}$, we shall mean that the nonwandering set of $f$ consists only of the hyperbolic orbits described by this data.

We construct first a diffeomorphism of a disc whose periodic points all have periods a power of 2 . This example is due to Dennis Pixton.

LEMMA 3.1. For any $n \geqslant 0$, there exists a diffeomorphism $f$ of the 2-dimensional unit disc onto its image with periodic data

$$
\left\{\left(2^{n+1}, 0,+1\right),(1,1,-1),(2,1,-1),\left(2^{2}, 1,-1\right), \ldots,\left(2^{n}, 1,-1\right)\right\} .
$$

Furthermore, for some $R>0, \varepsilon>0$, f maps the annulus $\left\{x \in R^{2}|1-R \leqslant| x \mid\right.$ $\leqslant 1\}$ inward along radii a fixed distance $\varepsilon$, and there exists a filtration of the disc for $f$.

Proof. Let $D_{0}^{1}$ denote the unit disc and let $D_{1}^{1}, D_{1}^{2}$ denote the two discs with radius $r_{1}=\frac{1}{4}$ and centers $a_{1}^{1}=\left(-\frac{1}{2}, 0\right), a_{1}^{2}=\left(\frac{1}{2}, 0\right)$; i.e., $D_{1}^{1}$ and $D_{1}^{2}$ are discs contained in $D_{0}^{1}$ with radii $\frac{1}{4}$ that of $D_{0}^{1}$ and centers on the $x$-axis at the midpoints of the two radii of $D_{0}^{1}$.

In general, for $2 \leqslant k \leqslant n+1$, let $D_{k}^{1}, D_{k}^{2}, \ldots, D_{k}^{2^{k}}$ denote the $2^{k}$ discs with radii $r_{k}=\frac{1}{4} r_{k-1}$ such that $D_{k}^{1}, D_{k}^{2} \subset D_{k-1}^{1} ; D_{k}^{3}, D_{k}^{4} \subset D_{k-1}^{2} ; \ldots ; D_{k}^{2^{k}-1}$, $D_{k}^{2^{k}} \subset D_{k-1}^{2^{k-1}}$, and such that the centers $a_{k}^{2 i-1}, a_{k}^{2 i}$ of $D_{k}^{2 i-1}, D_{k}^{2 i}$ respectively are the midpoints of the radii of $D_{k-1}^{i}$ on the $x$-axis. Choose $R>0$ small $\left(R<\frac{1}{16} r_{n+1}\right)$ and let $A_{0}^{1}$ be the annulus $\left\{x \in D_{0}^{1}\left|r_{0}-R \leqslant\right| x \mid \leqslant r_{0}\right\}$ and for $1 \leqslant k \leqslant n+1, A_{k}^{i}=\left\{x \in D_{0}^{1}\left|r_{k}-R \leqslant\right| x-a_{k}^{i} \mid \leqslant r_{k}+R\right\}$ (see Figure 1).

For $0 \leqslant k \leqslant n+1$, choose a $C^{\infty}$ function $\alpha_{k}: R \rightarrow[0,1]$ such that

$$
\alpha_{k}(t)= \begin{cases}1, & t \leqslant(7 / 8) r_{k} \\ 0, & (15 / 16) r_{k} \leqslant t .\end{cases}
$$

Now define $h_{k}: D_{k}^{1} \rightarrow D_{k}^{1}$ in polar coordinates centered at $a_{k}^{1}$ to be

$$
h_{k}(r, \theta)=\left(r, \theta+\pi \alpha_{k}(r)\right) \text {. }
$$

Then $h_{k}$ is a diffeomorphism of $D_{k}^{1}$ which is the identity on the annulus $A_{k}^{1} \cap D_{k}^{1}$ and is a rotation on the inner disc $\left\{x \in D_{k}^{1}|| x-a_{k}^{1} \mid<\frac{7}{8} r_{k}\right\}$ by $\pi$. 


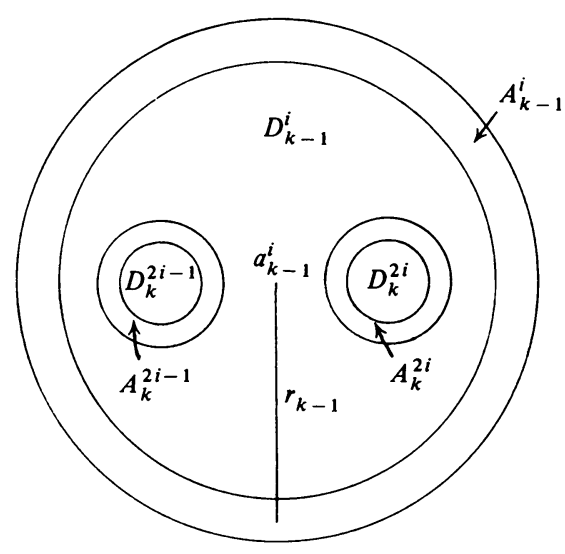

FIGURE 1

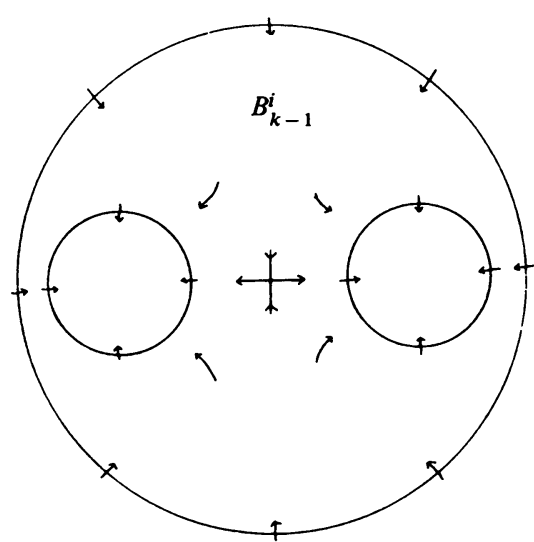

FIGURE 2

Note that the center of $D_{k}^{1}$ is a fixed point of $h_{k}$ and that $h_{k}$ reverses the two discs $D_{k+1}^{1}$ and $D_{k+1}^{2}$. In particular, $h_{k}$ interchanges the centers of these two discs. For $1 \leqslant k \leqslant n+1$, extend $h_{k}$ to the entire disc $D_{0}^{1}$-by the identity.

Define $h: D_{0}^{1} \rightarrow D_{0}^{1}$ by $h(x)=h_{0} \circ h_{1} \circ \ldots \circ h_{n+1}(x)$. Then $h$ is a diffeomorphism of $D_{0}^{1}$ with the following property: for each $k, 0<k<n+$ 1 , the centers of the $2^{k}$ discs $D_{k}^{i}$ form a periodic orbit of period $2^{k}$.

Let $B_{k}^{i}=D_{k}^{i} \cup A_{k}^{i}-\left\{\left(D_{k+1}^{2 i-1}-A_{k+1}^{2 i-1}\right) \cup\left(D_{k+1}^{2 i}-A_{k+1}^{2 i}\right)\right\}$, for $\mathrm{k}=0$, $1, \ldots, n$. Let $\rho_{k}$ be a flow on $\cup_{i=1}^{2^{k}} B_{k}^{i}$ with the following properties (Figure 2):

(a) $\rho_{k}$ maps the annuli $A_{k}^{i}, A_{k+1}^{2 i-1}$, and $A_{k+1}^{2 i}$ inward along radii a distance $\varepsilon$ at time 1;

(b) $\rho_{k}$ has exactly one saddle point at the center of each $B_{k}^{i}$;

(c) $\rho_{k}$ is symmetric in $B_{k}^{i}$ with respect to the center of $B_{k}^{i}$.

On the union of discs $\cup_{i=1}^{2^{n+1}}\left(D_{n+1}^{i} \cup A_{n+1}^{i}\right)$, define a flow $\rho_{n+1}$ with the properties:

(a) $\rho_{n+1}$ maps the annuli $A_{n+1}^{i}$ inward along radii a distance $\varepsilon$ at time 1;

(b) $\rho_{n+1}$ has exactly one sink at the center of each $D_{n+1}^{i}$;

(c) $\rho_{n+1}$ is symmetric in each $D_{n+1}^{i}$ with respect to the center of $D_{n+1}^{i}$.

The flows $\rho_{k}$ have been defined so that $\rho_{k}$ and $\rho_{k+1}$ agree where they are both defined, namely, on the annuli $A_{k+1}^{i}$. Therefore, we can define a flow $\rho$ on all of $D_{0}^{1}$ by setting $\rho(x)=\rho_{k}(x)$ for $x \in B_{k}^{i}$. Let $g$ be the time-one map of $\rho$. Then $g$ is a diffeomorphism of $D_{0}^{1}$ with $\sum_{i=0}^{n} 2^{i}$ saddle points of period 1 (the centers of the $D_{k}^{i}$ for $0 \leqslant k \leqslant n$ ) and $2^{n+1}$ sinks of period 1 (the centers of the $\left.D_{n+1}^{i}\right)$. All other points of $D_{0}^{1}$ are wandering under $g$.

Let $f=g \circ h$. Then $f$ is a diffeomorphism of $D_{0}^{1}$. For any $k, 0<k<n+$ 1 , the centers of the $2^{k}$ discs $D_{k}^{i}$ form a periodic orbit of period $2^{k}$ under $h$ and are left fixed by $g$. Thus, they form a periodic orbit of period $2^{k}$ under $f$. 
For each $k, 0 \leqslant k \leqslant n+1$, let $C_{k}^{i}=\left\{x \in D_{k}^{i}|| x-a_{k}^{i} \mid<\frac{1}{8} r_{k}\right\}$. Then $h$ permutes these $2^{k}$ discs, so for $x \in C_{k}^{i}, h(x) \in C_{k}^{j}$ for some $j$. Suppose $x=(r, \theta)$ in polar coordinates centered at $a_{k}^{i}$. Then $h(x)$ is either $(r, \theta)$ or $(r, \theta+\pi)$ in polar coordinates centered at $a_{k}^{j}$. But $\rho$ is symmetric with respect to the centers of the $D_{k}^{i}$. Hence $(g \circ h)(x)=(h \circ g)(x)$. Therefore, $\cup_{i=1}^{2^{k}} C_{k}^{i}$ is a neighborhood of the points $a_{k}^{i}$ in which $g$ and $h$ commute. Thus, in this neighborhood, $f^{2^{k}}=g^{2^{k}} \circ h^{2^{k}}$. Now, $h^{2^{k}}$ maps $C_{k}^{i}$ to itself (since $h^{2^{k}}\left(a_{k}^{i}\right)=a_{k}^{i}$ ), but $h^{2^{k}}$ interchanges the centers of the discs $D_{k+1}^{2 i-1}$ and $D_{k+1}^{2 i}$ (since they are points of period $2^{k+1}$ ). Therefore, $h^{2^{k}} \mid C_{k}^{i}$ is just rotation by $\pi$, and $D f^{2^{k}}=$ $D g^{2^{k}} \circ h$. Hence, the $n+1$ orbits consisting of the points $a_{k}^{i}$, for $0 \leqslant k \leqslant n$, are hyperbolic saddles and the orbit consisting of the $a_{n+1}^{i}$ is a hyperbolic sink. All other points are wandering under $f$.

Let $0 \leqslant k \leqslant n$, and let $W^{u}\left(a_{k}^{i}\right)$ denote the unstable manifold of $a_{k}^{i}$. We must show that $f^{2^{k}}$ reverses the orientation of $W^{u}\left(a_{k}^{i}\right)$. But in the neighborhood $C_{k}^{i}$ of $a_{k}^{i}, W^{u}\left(a_{k}^{i}\right)$ is on the $x$-axis, so its orientation is left fixed by $g^{2^{k}}$ and reversed by $h^{2^{k}}$, which rotates $C_{k}^{i}$ by $\pi$. Therefore, $f^{2^{k}}$ reverses the orientation of $W^{u}\left(a_{k}^{i}\right)$.

Note that $f$ maps the annulus $A_{0}^{1}$ inward along radii a distance $\varepsilon$, since this is the effect of $g$ and $h$ leaves $A_{0}^{1}$ fixed.

Finally, $f$ has the filtration

$$
D_{0}^{1} \supset D_{1}^{1} \cup D_{1}^{2} \supset \cdots \supset \bigcup_{i=1}^{2^{k}} D_{k}^{i} \supset \cdots \supset \bigcup_{i=1}^{2^{n+1}} D_{n+1}^{i} \supset \varnothing \text {. Q.E.D. }
$$

Lemma 3.2. Let $A=\left\{x \in \mathbf{R}^{2}|1 \leqslant| x \mid \leqslant 3\right\}$. For any $n \geqslant 1, m \geqslant 0$, there exists a diffeomorphism $f$ of $A$ onto its image with periodic data

$$
\left\{\left(2^{m} n, 0,+1\right),(n, 1,+1),(n, 1,-1),(2 n, 1,-1), \ldots,\left(2^{m-1} n, 1,-1\right)\right\} \text {. }
$$

Furthermore, for some $R>0, \varepsilon>0$, f maps the annuli

$$
A_{1}=\{x \in A|1 \leqslant| x \mid \leqslant 1+R\} \text { and } A_{2}=\{x \in A|3-R \leqslant| x \mid \leqslant 3\}
$$

into $A$ along radii a distance $\varepsilon$, and $f$ has a filtration on $A$.

Proof. Choose $R>0, \delta>0$ small, $0<R<\delta<1 / 3$ and let $\alpha: \mathbf{R} \rightarrow$ $[0,1]$ be a $C^{\infty}$ function such that

$$
\alpha(t)= \begin{cases}0, & t \leqslant 1+R \\ 1, & 2-\delta-R \leqslant t \leqslant 2+\delta+R, \\ 0, & 3-R \leqslant t .\end{cases}
$$

Define $h: A \rightarrow A$ in polar coordinates to be

$$
h(r, \theta)=\left(r, \theta+\frac{2 \pi}{n} \alpha(r)\right) .
$$

Then $h$ is a diffeomorphism of $A$ which is the identity on the annuli $A_{1}$ and 
$A_{2}$ and which rotates the annulus $\{x \in A|2-\delta-R<| x \mid<2+\delta+R\}$ by $2 \pi / n$.

Let $a_{k}=(2,2(k-1) \pi / n)$ for $k=1, \ldots, n$ and define sets $D_{k}=\left\{x \in A|| x-a_{k} \mid<\delta\right\}$ and $B_{k}=\left\{x \in A|\delta-R<| x-a_{k} \mid<\delta+R\right\}$ (see Figure 3).

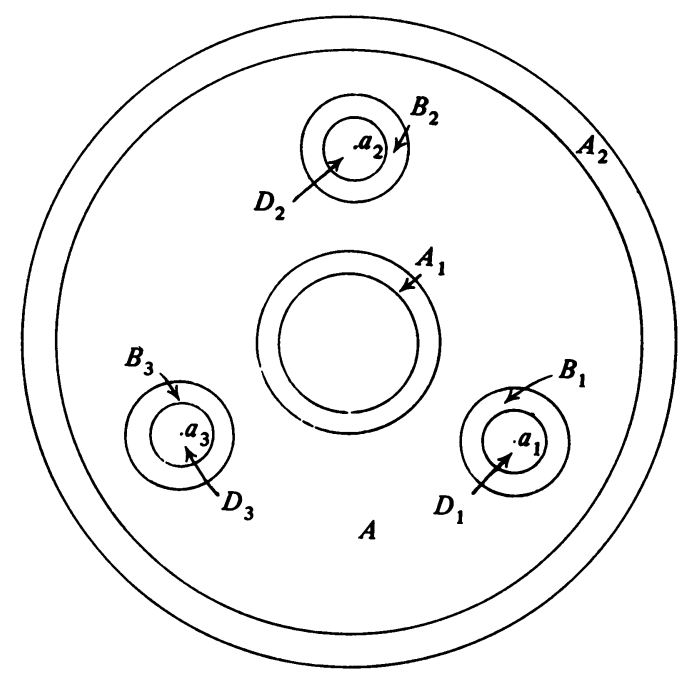

FIGURE 3

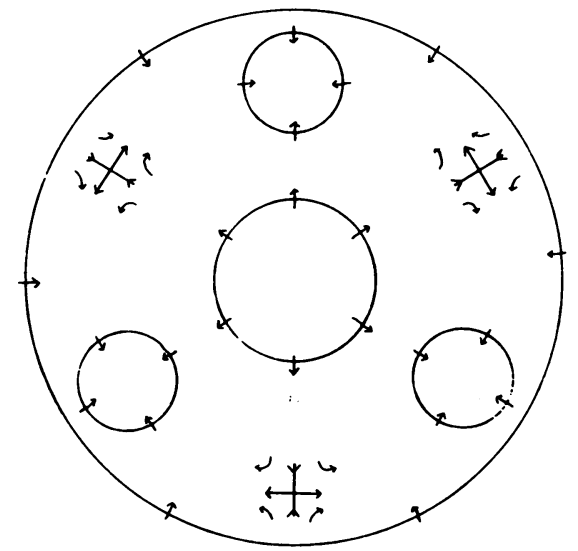

FIGURE 4

Let $B=A-\cup_{k=1}^{n}\left(D_{k}-B_{k}\right)$ and define a flow $\rho$ on $B$ with the following properties (Figure 4):

(a) $\rho$ maps the annuli $A_{1}$ and $A_{2}$ into $A$ along radii a distance of $\varepsilon$ at time 1; 
(b) $\rho$ maps the $n$ annuli $B_{k}$ inward along radii a distance of $\varepsilon$ at time 1;

(c) $\rho$ has $n$ saddle points at $b_{k}=(2,(2 k-1) \pi / n), k=1, \ldots, n$ and all other points are wandering;

(d) $\rho$ commutes with rotation through $2 \pi / n$.

Let $\varphi$ be the time-one map of $\rho$. Then $\varphi$ has $n$ saddles of orientation type +1 .

We shall now define diffeomorphisms $g_{1}, \ldots, g_{n}$ on the discs $D_{1} \cup$ $B_{1}, \ldots, D_{n} \cup B_{n}$. In each disc $D_{k} \cup B_{k}$, choose the diametral axis which makes an angle of $2(k-1) \pi / n$ with the positive $x$-axis. By Lemma 3.1, there exists a diffeomorphism $g_{1}$ of $D_{1} \cup B_{1}$ with $m$ saddles of periods $1,2, \ldots, 2^{m-1}$ respectively and each of orientation type -1 , and one sink of period $2^{m}$, and such that the annulus $B_{1}$ is mapped inward along radii a distance $\varepsilon$. Let $c_{1}^{i}$ denote a point of period $2^{i}$. Then, as is clear from the proof of Lemma 3.1, we can choose these $\sum_{i=0}^{m} 2^{i}$ points so that they all lie on the given axis of $D_{1} \cup B_{1}$. Now for $2 \leqslant k \leqslant n$, define a flow $\rho_{k}$ on $D_{k} \cup B_{k}$ with the following properties (Figure 5):

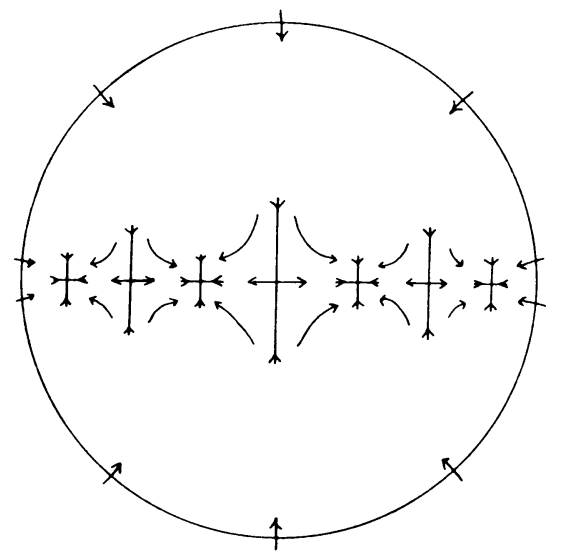

FIGURE 5

(a) $\rho_{k}$ maps the annulus $B_{k}$ inward along radii a distance $\varepsilon$ at time 1;

(b) at each of the $\sum_{i=0}^{m-1} 2^{i}$ points $c_{k}^{i}$ on the given axis such that $\left|c_{k}^{i}-a_{k}\right|=$ $\left|c_{1}^{i}-a_{1}\right|, \rho_{k}$ has a saddle point;

(c) at each of the $2^{m}$ points $c_{k}^{m}$ such that $\left|c_{k}^{m}-a_{k}\right|=\left|c_{1}^{m}-a_{1}\right|, \rho_{k}$ has a sink;

(d) all other points are wandering under $\rho_{k}$.

Let $g_{k}$ be the time-one map of $\rho_{k}, k=2, \ldots, n$. Now define a diffeomorphism $g$ of $A$ by

$$
g(x)= \begin{cases}\varphi(x), & x \in B, \\ g_{i}(x), & x \in D_{i} \cup B_{i} .\end{cases}
$$

Then $g$ is well defined, since $\varphi$ and $g_{i}$ agree on $B \cap\left(D_{i} \cup B_{i}\right)=B_{i}$. 
Let $f=g \circ h$. The points $b_{1}, \ldots, b_{n}$ are saddle points of $g$ and are permuted by $h$. Also, in the neighborhood $\cup_{i=1}^{n} D_{i}^{\prime}, D_{i}^{\prime}=\left\{x \in A|| x-b_{i} \mid\right.$ $<\delta\}, g$ and $h$ commute and $h \mid \cup D_{i}^{\prime}$ is just rotation by $2 \pi / n$. Therefore, $D f^{n}=D g^{n} \circ h$, so the points $b_{1}, \ldots, b_{n}$ form a hyperbolic saddle of period $n$. Furthermore, both $h$ and $g$ leave the orientation of $W^{u}\left(b_{i}\right)$ fixed for all $i$, so this saddle has orientation type +1 .

Now consider the points $c_{j}^{i}$. Under $g$, the $\sum_{i=0}^{m} 2^{i}$ points $c_{1}^{i}$ form $m+1$ hyperbolic orbits: $m$ saddles of periods $1,2, \ldots, 2^{m-1}$ respectively and orientation type -1 , and 1 sink of period $2^{m}$. For $j>1$, the $c_{j}^{i}$ are left fixed by $g$. For each $i, h^{k}\left(c_{1}^{i}\right)=c_{k+1}^{i}, 1 \leqslant k \leqslant n-1$, and $h^{n}\left(c_{1}^{i}\right)=c_{1}^{i}$. Therefore, $f$ has the following periodic orbits: $m$ saddles of periods $n, 2 n, \ldots, 2^{m-1} n$ respectively, and one sink of period $2^{m} n$. As in the proof of Lemma 3.1, there exists a small disc $C_{1}^{i}$ about each $c_{1}^{i}$ such that on $\cup_{i} C_{1}^{i}$, the mappings $g$ and $h$ commute. Furthermore, these $C_{1}^{i}$ may be chosen so that $h^{k} \mid C_{1}^{i}$ is just rotation in coordinates centered at 0 by $2 k \pi / n$. Therefore, in the neighborhood

$$
\bigcup_{i} C_{1}^{i} \cup \bigcup_{k, i} h^{k}\left(C_{1}^{i}\right)
$$

of the $c_{j}^{i}, g$ and $h$ commute, and $D f^{k}=D g^{k} \circ h, 1 \leqslant k \leqslant 2^{m} n$. Hence, these orbits are hyperbolic orbits. Finally, it is clear that the orientation type of the saddles formed this way is -1 , since rotation of $A$ by $h$ does not change the orientation of their unstable manifolds.

That $f$ acts as desired in a neighborhood of the boundary of $A$ is clear from the construction.

Let $D_{k} \supset M_{k}^{1} \supset M_{k}^{2} \supset \cdots \supset M_{k}^{\nu_{k}} \supset \varnothing$ be the filtration for $f \mid D_{k}$ as described in Lemma 3.1. Then a filtration for $f$ is given by

$$
A \supset \bigcup_{k=1}^{n} D_{k} \supset \bigcup_{k=1}^{n} M_{k}^{1} \supset \cdots \supset \bigcup_{k=1}^{n} M_{k}^{\nu_{k}} \supset \varnothing \text {. Q.E.D. }
$$

LemmA 3.3. Let $A, A_{1}$, and $A_{2}$ be as in Lemma 3.2. For any $n \geqslant 1, m \geqslant 0$, there exists a diffeomorphism $f$ of $A$ onto its image with periodic data

$$
\left\{\left(2^{m} n, 0,+1\right),(n, 1,+1),(n, 1,-1),(2 n, 1,-1), \ldots,\left(2^{m-1} n, 1,-1\right)\right\}
$$

and such that $f$ maps the annulus $A_{1}$ along radii a distance $\varepsilon$ outside of $A$ and the annulus $A_{2}$ a distance $\varepsilon$ into $A$. Furthermore, there exist submanifolds

$$
A \supset N_{k} \supset N_{k-1} \supset \cdots \supset N_{1} \supset \varnothing
$$

such that $f\left(N_{i}\right) \subset$ int $N_{i}$ and $\cap{ }_{m \in \mathbf{Z}} f^{m}\left(N_{i}-N_{i-1}\right), i=1, \ldots, k$, and $\cap_{m \in \mathbf{Z}} f^{m}\left(A-N_{k}\right)$ are the periodic orbits of $f$.

Proof. The proof of this lemma is almost identical to that of Lemma 3.2, so we shall use the notation of that proof and simply indicate the necessary changes. This consists mainly of changing the definition of the flow $\rho$ defined 
on the annulus $A$ with discs $\left\{x \in A|| x-a_{k} \mid<\delta-R\right\}$ removed.

Let $b_{k}=(3 / 2,2(k-1) \pi / n)$ and $D_{k}^{\prime}=\left\{x \in A|| x-b_{k} \mid<\delta\right\}$. Note that $\delta$ and $R$ may be chosen as small as desired, so that $D_{k}^{\prime} \cap\left(D_{k} \cup B_{k}\right)=\varnothing$ and $D_{k}^{\prime} \cap A_{1}=\varnothing$. Define a flow $\rho$ on $B=A-\cup_{k=1}^{n}\left(D_{k}-B_{k}\right)$ with the following properties (Figure 6):

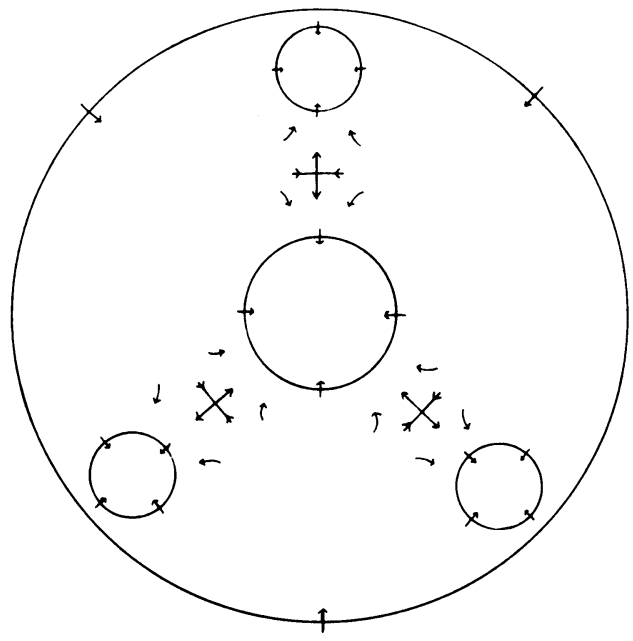

FIGURE 6

(a) $\rho$ maps the annulus $A_{1}$ along radii outside of $A$ a distance $\varepsilon$ at time 1, and $\rho$ maps $A_{2}$ along radii into $A$ a distance $\varepsilon$ at time 1;

(b) $\rho$ maps the $n$ annuli $B_{k}$ inward along radii a distance of $\varepsilon$ at time 1;

(c) $\rho$ has $n$ saddle points at $b_{1}, \ldots, b_{n}$ and all other points are wandering;

(d) $\rho$ commutes with rotation through $2 \pi / n$.

If $f$ is defined as in 3.2, then $f$ is a diffeomorphism with domain $A$ which has the same periodic data as the diffeomorphism in 3.2 and differs only in that it maps a neighborhood of the inner boundary of $A$ outside of $A$ instead of into A.

Finally, $A \supset \cup_{k=1}^{n} D_{k} \supset \cup_{k=1}^{n} M_{k}^{1} \supset \cdots \supset \cup_{k=1}^{n} M_{k}^{\nu_{k}} \supset \varnothing$, where the $M_{k}^{i}$ are as in 3.2, and these submanifolds satisfy the properties in the statement of the lemma. Q.E.D.

In the proof of the theorem, we shall write the 2-manifold $M$ as a union of annuli and discs, $\cup A_{i}$, and construct $f \in \operatorname{Diff}(M)$ such that $f \mid A_{i}$ is one of the examples constructed above. These submanifolds will be pieced together so that they would give a filtration for $f$ if $f \mid A_{i}$ had only one periodic orbit. On the other hand, as seen in the preceding lemmas, $f \mid A_{i}$ would have a filtration on $A_{i}$ if $f\left(A_{i}\right)$ were always contained in $A_{i}$. We can combine these subdivisions to get a filtration for $f$, and we state the following as a lemma for purposes of reference. 
LEMMA 3.4. Let $f \in \operatorname{Diff}(M)$ and suppose there exist submanifolds $M_{1}, \ldots, M_{k}$ and $N_{1}^{1}, \ldots, N_{\nu_{1}}^{1}, \ldots, N_{1}^{k}, \ldots, N_{\nu_{k}}^{k}$ of $M$, with boundary and of the same dimension as $M$, satisfying

(1) $M=M_{k} \supset M_{k-1} \supset \cdots \supset M_{1} \supset \varnothing$ and $f\left(M_{i}\right) \subset$ int $M_{i}$;

(2) for $i=1, \ldots, k, M_{i}-M_{i-1}=N_{\nu_{i}}^{i} \supset \cdots \supset N_{1}^{i} \supset \varnothing$ and

$$
\begin{gathered}
f\left(N_{j}^{i}\right) \subset \text { int } N_{j}^{i}, \quad j=1, \ldots, \nu_{i}-1, \\
\bigcap_{m \in \mathbf{Z}} f^{m}\left(N_{j}^{i}-N_{j-1}^{i}\right)=\Lambda_{j}^{i}, \quad j=1, \ldots, \nu_{i},
\end{gathered}
$$

where $\left\{\Lambda_{j}^{i}\right\}_{i j}$ are the basic sets of $f$. Then $f$ has a filtration on $M$.

Proof. We have

$$
\begin{aligned}
M & =M_{k}=M_{k-1} \cup N_{v_{k}}^{k} \supset \cdots \supset M_{k-1} \cup N_{1}^{k} \supset M_{k-1} \\
& =M_{k-2} \cup N_{\nu_{k-1}}^{k-1} \supset \cdots \supset M_{2}=M_{1} \cup N_{\nu_{2}}^{2} \supset \cdots \supset M_{1} \cup N_{1}^{2} \supset M_{1} \\
& =N_{\nu_{1}}^{1} \supset \cdots \supset N_{1}^{1} \supset \varnothing,
\end{aligned}
$$

where $f\left(M_{j} \cup N_{i}^{j+1}\right) \subset \operatorname{int}\left(M_{j} \cup N_{i}^{j+1}\right)$ and

$$
\bigcap_{m \in \mathbf{Z}} f^{m}\left[\left(M_{j} \cup N_{i}^{j+1}\right)-\left(M_{j} \cup N_{i-1}^{j+1}\right)\right]=\bigcap_{m \in \mathbf{Z}} f^{m}\left(N_{i}^{j+1}-N_{i-1}^{j+1}\right)=\Lambda_{i}^{j+1},
$$

for all $i, j$. Q.E.D.

We shall now prove that the conditions of the theorem are sufficient when $M$ is a compact, connected 2-manifold. By the classification theorem for compact surfaces (see Massey [4] for a reference), $M$ is homeomorphic to a sphere, torus, connected sum of tori, projective plane, or connected sum of projective planes. We shall denote these surfaces by $S^{2}, T, X_{n}, P$, and $Y_{n}$ respectively.

THEOREM. Let $M$ be a compact, connected 2-manifold. There exists a Morse-Smale diffeomorphism $f$ of $\boldsymbol{M}$ homotopic to the identity with periodic data $\left\{\left(p_{i}, u_{i}, \Delta_{i}\right)\right\}$ if

(a) $u_{i}=0$ and $u_{j}=2$ for some $i$ and $j$;

(b) $\prod_{k=1}^{n}\left(1-\Delta_{i} t^{p_{i}}\right)^{(-1)^{u_{i}}}=(1-t)^{x(M)}$, where $n$ is the number of periodic orbits.

Proof. Since $M$ is a 2-dimensional manifold, $u_{i}$ is 0,1 , or 2 depending on whether $\gamma_{i}$ is a sink, saddle, or source. Furthermore, in the case of a diffeomorphism homotopic to the identity, $\Delta_{i}$ is +1 when $\gamma_{i}$ is a sink or source, and may be +1 or -1 when $\gamma_{i}$ is a saddle. Assume that the periodic data is

$$
\begin{aligned}
\left\{\left(p_{1}, u_{1},+1\right), \ldots,\left(p_{m}, u_{m},+1\right),\left(q_{1}, 1,+1\right), \ldots,\right. \\
\left.\left(q_{l}, 1,+1\right),\left(r_{1}, 1,-1\right), \ldots,\left(r_{s}, 1,-1\right)\right\},
\end{aligned}
$$


where $u_{i}=2$ for $k$ indices $i$ and $u_{i}=0$ for $m-k$ indices; that is, the periodic orbits consist of $k$ sources and $m-k$ sinks of periods $p_{1}, \ldots, p_{m} ; l$ saddles of periods $q_{1}, \ldots, q_{l}$ and orientation type +1 ; and $s$ saddles of periods $r_{1}, \ldots, r_{s}$ and orientation type -1 . Then by condition (a), $k \geqslant 1$ and $m-k \geqslant 1$, and by condition (b),

$$
\frac{(1-t)^{\gamma(M)} \Pi_{i=1}^{l}\left(1-t^{q_{i}}\right) \Pi_{i=1}^{s}\left(1+t^{r_{i}}\right)}{\Pi_{j=1}^{m}\left(1-t^{p_{j}}\right)}=1 .
$$

We shall consider the case of each compact, connected 2-manifold separately. (1) $M=S^{2}$. If $M=S^{2}$, then $\chi(M)=2$. After reindexing the $q$ 's, we can write the equation $(*)$ as

$$
\frac{\prod_{i=1}^{l+2}\left(1-t^{q_{i}}\right) \prod_{i=1}^{s}\left(1+t^{r_{i}}\right)}{\prod_{j=1}^{m}\left(1-t^{p_{j}}\right)}=1, \text { where } q_{1}=q_{l+2}=1 .
$$

By Lemma 2.1, $m=l+2$ and there exist integers $a_{1} \geqslant 0, \ldots, a_{m}>0$ such that the set $\left\{p_{1}, \ldots, p_{m}\right\}$ equals the set $\left\{2^{a_{1}} q_{1}, \ldots, 2^{a_{m}} q_{m}\right\}$ and the set $\left\{r_{1}, \ldots, r_{s}\right\}$ equals the set

$$
\left\{q_{1}, 2 q_{1}, \ldots, 2^{a_{1}-1} q_{1} ; \cdots ; q_{m}, 2 q_{m}, \ldots, 2^{a_{m}-1} q_{m}\right\} .
$$

Note that in this notation, the equation is

$$
\prod_{k=1}^{m}\left[\frac{\left(1-t^{q_{i}}\right)\left(1+t^{q_{i}}\right) \cdots\left(1+t^{2^{a_{i-1}} q_{i}}\right)}{\left(1-t^{2^{2 i} q_{i}}\right)}\right]=1 .
$$

We see that there is a natural grouping of the data for sources and sinks with the data for saddles. In fact, we can rewrite the periodic data now as

$$
\begin{aligned}
& \left\{\left(2^{a_{1}}, u_{1},+1\right),(1,1,-1), \ldots,\left(2^{a_{1}-1}, 1,-1\right) ;\right. \\
& \left(2^{a_{m}}, u_{m},+1\right),(1,1,-1), \ldots,\left(2^{a_{m}-1}, 1,-1\right) ; \\
& \left(2^{a_{i}} q_{i}, u_{i},+1\right),\left(q_{i}, 1,+1\right),\left(q_{i}, 1,-1\right), \ldots, \\
& \left.\left(2^{a_{i}-1} q_{i}, 1,-1\right), i=2, \ldots, m-1\right\}
\end{aligned}
$$

and the idea of the proof is to construct $m$ diffeomorphisms on subsets of $S^{2}$, each of which contributes one block to the data set.

Write $S^{2}$ as a union $S^{2}=A_{1} \cup \cdots \cup A_{m}$, where $A_{1}$ and $A_{m}$ are 2dimensional discs, $A_{2}, \ldots, A_{m-1}$ are 2-dimensional annuli, and the sets overlap in the following way: $A_{i} \cap A_{i+1}, i=1, \ldots, m-1$, are annuli of width $R$ (where $R$ is small compared to the diameters of the discs and the widths of the annuli), and all other intersections are empty. We shall construct a diffeomorphism of $S^{2}$ by piecing together suitable diffeomorphisms $f_{i}$ defined on $A_{i}$. The $f_{i}$ are all special cases of the mappings constructed in Lemmas 3.1, 3.2, 3.3 or their inverses. Thus, each such $f_{i}$ will 
have the property that it maps annuli of width $R$ containing $\partial A_{i} \cap A_{i-1}$ and $\partial A_{i} \cap A_{i+1}$ along normals to $\partial A_{i}$ a fixed distance $\varepsilon$, where $\varepsilon<R$, either outside $A_{i}$ or into $A_{i}$. Therefore, in piecing together the $f_{i}$, the only thing we must be sure of is that $f_{i}$ and $f_{i+1}$ both map $A_{i} \cap A_{i+1}$ in the same direction. Below, we shall simply indicate in each case whether $f_{i}$ maps $\partial A_{i} \cap A_{i-1}$ outside or into $A_{i}$ and $\partial A_{i} \cap A_{i+1}$ outside or into $A_{i}$.

Assume that the $p$ 's are indexed so that $p_{i}=2^{a_{i}} q_{i}$. Then $p_{1}=2^{a_{1}}, p_{m}=2^{a_{m}}$ and there are three possibilities: $2^{a_{1}}$ and $2^{a_{m}}$ are periods of two sources, two sinks, or one source and one sink.

(i) Suppose that $p_{1}$ is the period of a source and $p_{m}$ is the period of a sink (that is, we have $\left(2^{a_{1}}, 2,+1\right)$ and $\left(2^{a_{m}}, 0,+1\right)$ in the given data). Let $f_{1}$ be a diffeomorphism of $A_{1}$ onto its image with periodic data

$$
\left\{\left(2^{a_{1}}, 2,+1\right),(1,1,-1),(2,1,-1), \ldots,\left(2^{a_{1}-1}, 1,-1\right)\right\}
$$

and which maps $\partial A_{1}$ out of $A_{1}$. Such a diffeomorphism exists by Lemma 3.1 (taking inverses).

Also by 3.1 , there exists a diffeomorphism $f_{m}$ of $A_{m}$ onto its image with periodic data

$$
\left\{\left(2^{a_{m}}, 0,+1\right),(1,1,-1),(2,1,-1), \ldots,\left(2^{a_{m}-1}, 1,-1\right)\right\}
$$

and which maps $\partial A_{m}$ into $A_{m}$.

Now we have one orbit which is a source and one which is a sink, and $m-2$ such orbits left to define, $k-1$ sources and $m-k-1$ sinks (where $k-1$ and $m-k-1$ may be zero). Without loss of generality, we may assume that the $p$ 's are indexed so that

$$
p_{2}=2^{a_{2}} q_{2}, \ldots, p_{k}=2^{a_{k}} q_{k}
$$

are periods of sources and $p_{k+1}=2^{a_{k+1}} q_{k+1}, \ldots, p_{m-1}=2^{a_{m-1}} q_{m-1}$ are periods of sinks. By Lemma 3.3 (taking inverses), there exist diffeomorphisms $f_{2}, \ldots, f_{k}$ with domains $A_{2}, \ldots, A_{k}$ respectively, such that $f_{i}$ has periodic data

$$
\begin{array}{r}
\left\{\left(2^{a_{i}} q_{i}, 2,+1\right),\left(q_{i}, 1,+1\right),\left(q_{i}, 1,-1\right),\right. \\
\left.\left(2 q_{i}, 1,-1\right), \ldots,\left(2^{a_{i}-1} q_{i}, 1,-1\right)\right\}
\end{array}
$$

and such that $f_{i}$ maps $\partial\left(A_{i}\right) \cap A_{i-1}$ into $A_{i}$ and $\partial\left(A_{i}\right) \cap A_{i+1}$ out of $A_{i}$.

Also by 3.3 , there exist diffeomorphisms $f_{k+1}, \ldots, f_{m-1}$ with domains $A_{k+1}, \ldots, A_{m-1}$ respectively such that $f_{j}$ has periodic data

$$
\begin{array}{r}
\left\{\left(2^{a_{j}} q_{j}, 0,+1\right),\left(q_{j}, 1,+1\right),\left(q_{j}, 1,-1\right),\right. \\
\left.\left(2 q_{j}, 1,-1\right), \ldots,\left(2^{a_{j}-1} q_{j}, 1,-1\right)\right\}
\end{array}
$$

and $f_{j}$ maps $\partial\left(A_{j}\right) \cap A_{j-1}$ into $A_{j}$ and $\partial\left(A_{j}\right) \cap A_{j+1}$ out of $A_{j}$ (see Figure 7). 


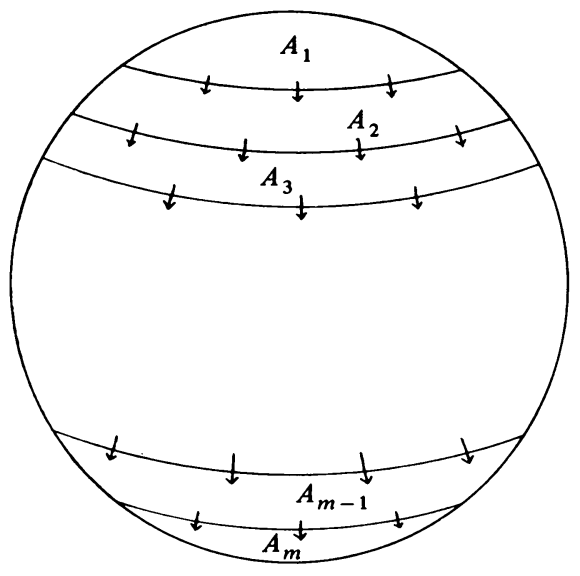

FigURE 7

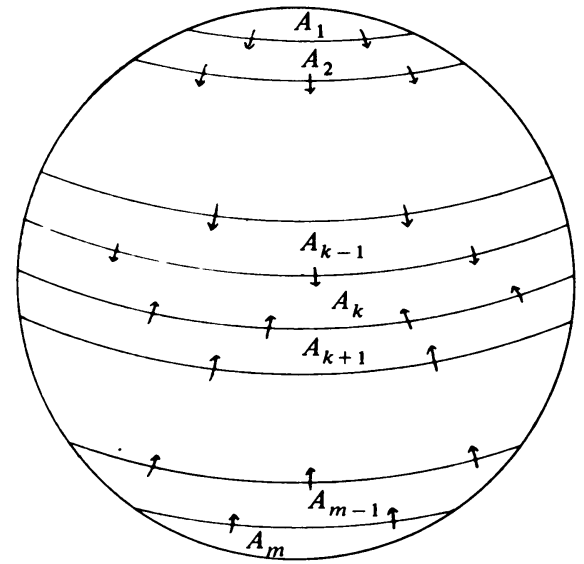

FigURE 8

Since $f_{i}$ and $f_{i+1}$ agree on $A_{i} \cap A_{i+1}, i=1, \ldots, m-1$, we can define a diffeomorphism $F$ of $S^{2}$ by $F(x)=f_{i}(x), x \in A_{i}$. Then the periodic data of $F$ is the union of the sets of periodic data of the $f_{i}$. Therefore, $F$ has the given periodic data.

Let $N_{i}=\cup_{j=i}^{m} A_{j}$. Then $S^{2}=N_{1} \supset N_{2} \supset \cdots \supset N_{m} \supset \varnothing, \quad F\left(N_{i}\right) \subset$ int $N_{i}$, and for $i=1, \ldots, m, N_{i}-N_{i-1}=A_{i}$ satisfies condition (2) of Lemma 3.4. Therefore, $F$ has a filtration on $S^{2}$. By the $\Omega$-stability theorem [10], there exists a neighborhood $N$ of $F$ such that any $h \in N$ has the same periodic data as $F$. By the Kupka-Smale theorem [1], [7], there exists $f \in N$ such that $f$ satisfies the transversal intersection condition. Thus, after a small perturbation of $F$, we have a Morse-Smale diffeomorphism $f$ of $S^{2}$ with the given periodic data.

(ii) Now suppose that $p_{1}$ and $p_{m}$ are both periods of sources. Let $g_{1}=f_{1}$ and $g_{m}=f_{m}^{-1}$, where $f_{1}$ and $f_{m}$ are defined in (i).

Then $g_{1}$ and $g_{m}$ have domains $A_{1}$ and $A_{m}$ respectively, $g_{i}$ maps a neighborhood of $\partial A_{i}$ out of $A_{i}$, and $g_{i}$ has periodic data

$$
\left\{\left(2^{a_{1}}, 2,+1\right),(1,1,-1),(2,1,-1), \ldots,\left(2^{a_{i}-1}, 1,-1\right)\right\}
$$

for $i=1$ and $i=m$.

Assume that the $p$ 's are indexed so that $p_{2}, \ldots, p_{k-1}$ are periods of sources and $p_{k}, \ldots, p_{m-1}$ are periods of sinks. Note that $k-1$ may be zero, but by condition (a) of the theorem, there must be at least one sink, so $m-1 \geqslant k$. Let $g_{i}=f_{i}$ for $i=2, \ldots, k-1$, where $f_{i}$ is defined in (i). Then $g_{i}$ has domain $A_{i}, g_{i}$ maps $\partial A_{i} \cap A_{i-1}$ into $A_{i}$ and $\partial A_{i} \cap A_{i+1}$ out of $A_{i}$, and $g_{i}$ has periodic 
data

$$
\begin{array}{r}
\left\{\left(2^{a_{i}} q_{i}, 2,+1\right),\left(q_{i}, 1,+1\right),\left(q_{i}, 1,-1\right),\left(2 q_{i}, 1,-1\right), \ldots,\right. \\
\left.\left(2^{a_{i}-1} q_{i}, 1,-1\right)\right\} .
\end{array}
$$

By Lemma 3.2, there exists a diffeomorphism $g_{k}$ of $A_{k}$ onto its image such that $g_{k}$ maps both $\partial A_{k} \cap A_{k-1}$ and $\partial A_{k} \cap A_{k+1}$ into $A_{k}$ and such that $g_{k}$ has periodic data

$$
\left\{\left(2^{a_{k}} q_{k}, 0,+1\right),\left(q_{k}, 1 ;+1\right),\left(q_{k}, 1,-1\right), \ldots,\left(2^{a_{k}-1} q_{k}, 1,-1\right)\right\} .
$$

Finally, let $g_{k+1}, \ldots, g_{m-1}$ be diffeomorphisms with domains $A_{k+1}, \ldots, A_{m-1}$ respectively such that for $i=k+1, \ldots, m-1, g_{i}$ has periodic data

$$
\left\{\left(2^{a_{i}} q_{i}, 0,+1\right),\left(q_{i}, 1,+1\right),\left(q_{i}, 1,-1\right), \ldots,\left(2^{a_{i}-1} q_{i}, 1,-1\right)\right\}
$$

and $g_{i}$ maps $\partial A_{i} \cap A_{i-1}$ outside of $A_{i}$ and $\partial A_{i} \cap A_{i+1}$ into $A_{i}$ (see Figure 8). Since $g_{i}$ and $g_{i+1}$ agree on $A_{i} \cap A_{i+1}$, we can define a diffeomorphism $g$ of $S^{2}$ by $g(x)=g_{i}(x), x \in A_{i}$, and $g$ has the given periodic data.

Let

$$
N_{i}= \begin{cases}\cup_{j=i}^{m} A_{j}, & i=1, \ldots, k, \\ \bigcup_{j=k}^{m+k-j} A_{j}, & j=k+1, \ldots, m .\end{cases}
$$

Then $S^{2}=N_{1} \supset N_{2} \supset \cdots \supset N_{m} \supset \varnothing$, and $g\left(N_{i}\right) \subset \operatorname{int}\left(N_{i}\right)$ for each $i$. Also, $N_{i}-N_{i-1}$ satisfies the conditions of Lemma 3.4 for each $i$, so $g$ has a filtration on $S^{2}$. Therefore $g$ is $\Omega$-stable, and there exists a diffeomorphism $f$ of $S^{2}$ which is topologically conjugate to $g$ on $\Omega$ and which has transversal intersections. Then $f$ is a Morse-Smale diffeomorphism of $S^{2}$ with the given periodic data.

(iii) The case when $p_{1}$ and $p_{m}$ are both periods of sinks follows immediately from (ii) by taking the inverse of $g$ (after suitable reindexing of the $p$ 's).

(2) $M=T$. In this case, $\chi(M)=0$ and the canonical form of the equation (*) is

$$
\prod_{i=1}^{m}\left[\frac{\left(1-t^{q_{i}}\right)\left(1+t^{q_{i}}\right)\left(1+t^{2 q_{i}}\right) \cdots\left(1+t^{2^{a_{i}-1} q_{i}}\right)}{\left(1-t^{2^{a_{i}} q_{i}}\right)}\right]=1,
$$

where there are no restrictions on the $q_{i}$.

Write $T$ as a union $T=A_{1} \cup \cdots \cup A_{m}$, where the $A_{i}$ are 2-dimensional annuli such that $A_{i} \cap A_{i+1}, i=1, \ldots, m-1$, and $A_{m} \cap A_{1}$ are annuli of width $R$ (where $R$ is small as above), with all other intersections empty.

Assume that $p_{1}=2^{a_{1}} q_{1}, \ldots, p_{k}=2^{a_{k}} q_{k}$ are periods of sources and $p_{k+1}=$ $2^{a_{k+1}} q_{k+1}, \ldots, p_{m}=2^{a_{m}} q_{m}$ are periods of sinks. By condition (a) of the theorem, $k \geqslant 1, m-k \geqslant 1$. 
By Lemma 3.2, there exists a diffeomorphism $f_{1}$ with domain $A_{1}$ such that $f_{1}$ has periodic data

$$
\left\{\left(2^{a_{1}} q_{1}, 2,1\right),\left(q_{1}, 1,1\right),\left(q_{1}, 1,-1\right), \ldots,\left(2^{a_{1}-1} q_{1}, 1,-1\right)\right\},
$$

and such that $f_{1}$ maps the boundaries $\partial A_{1} \cap A_{m}$ and $\partial A_{1} \cap A_{2}$ out of $A_{1}$. Also by 3.2 , there exists a diffeomorphism $f_{k+1}$ defined on $A_{k+1}$ with periodic data

$$
\begin{array}{r}
\left\{\left(2^{a_{k+1}} q_{k+1}, 0,1\right),\left(q_{k+1}, 1,1\right),\left(q_{k+1}, 1,-1\right), \ldots,\right. \\
\left.\left(2^{a_{k+1}-1} q_{k+1}, 1,-1\right)\right\}
\end{array}
$$

and such that $f_{k+1}$ maps the boundaries $\partial A_{k+1} \cap A_{k}$ and $\partial A_{k+1} \cap A_{k+2}$ into $A_{k+1}$. Now let $f_{2}, \ldots, f_{k}, f_{k+2}, \ldots, f_{m}$ be diffeomorphisms defined on $A_{2}, \ldots, A_{k}, A_{k+2}, \ldots, A_{m}$ respectively with the following properties:

(i) for $i=2, \ldots, k, f_{i}$ has periodic data

$$
\left\{\left(2^{a_{i}} q_{i}, 2,+1\right),\left(q_{i}, 1,+1\right),\left(q_{i}, 1,-1\right), \ldots,\left(2^{a_{i}-1} q_{i}, 1,-1\right)\right\}
$$

and $f_{i}$ maps $\partial A_{i} \cap A_{i-1}$ into $A_{i}$ and $\partial A_{i} \cap A_{i+1}$ out of $A_{i}$;

(ii) for $i=k+2, \ldots, m, f_{i}$ has periodic data

$$
\left\{\left(2^{a_{i}} q_{i}, 0,+1\right),\left(q_{i}, 1,+1\right),\left(q_{i}, 1,-1\right), \ldots,\left(2^{a_{i}-1} q_{i}, 1,-1\right)\right\}
$$

and $f_{i}$ maps $\partial A_{i} \cap A_{i-1}$ out of $A_{i}$ and $\partial A_{i} \cap A_{i+1}\left(\partial A_{m} \cap A_{1}\right)$ into $A_{i}$ (see Figure 9).

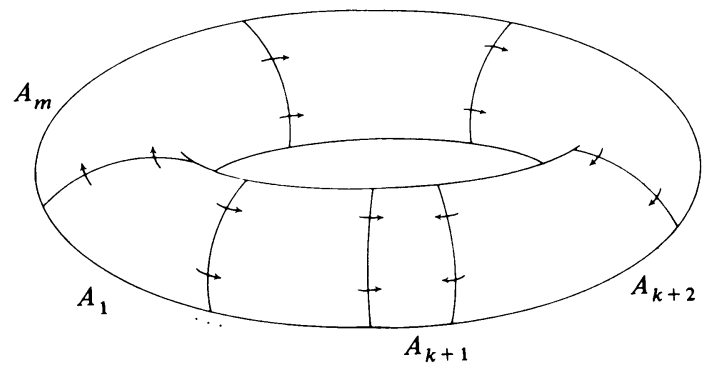

FiguRE 9

Such diffeomorphisms exist by Lemma 3.3.

Let $F(x)=f_{i}(x), x \in A_{i}$. The $f_{i}$ are constructed so that $F$ is well defined. Then $F$ is a diffeomorphism of $T$ with periodic data

$$
\begin{array}{r}
\left\{\left(2^{a_{i}} q_{i}, 2,+1\right),\left(q_{i}, 1,+1\right),\left(q_{i}, 1,-1\right), \ldots,\left(2^{a_{i}-1} q_{i}, 1,-1\right)\right. \\
\left(2^{a_{j}} q_{j}, 0,+1\right),\left(q_{j}, 1,+1\right),\left(q_{j}, 1,-1\right), \ldots,\left(2^{a_{j}-1} q_{j}, 1,-1\right) ; \\
i=1, \ldots, k, j=k+1, \ldots, m\} .
\end{array}
$$


Now define sets

$$
N_{i}= \begin{cases}\cup_{j=i}^{m} A_{j}, & i=1, \ldots, k+1, \\ \bigcup_{j=k+1}^{m+k+1-j} A_{j}, & i=k+2, \ldots, m .\end{cases}
$$

Then $T=N_{1} \supset N_{2} \supset \cdots \supset N_{m} \supset \varnothing, F\left(N_{i}\right) \subset \operatorname{int}\left(N_{i}\right)$, and the $N_{i}-N_{i-1}$ satisfy the conditions of Lemma 3.4. Hence, $F$ has a filtration on $T$ and is $\Omega$-stable. Then there exists a diffeomorphism $f$ of $T$ with the same periodic data as $F$ and which satisfies the transversal intersection condition. Therefore, $f$ is a Morse-Smale diffeomorphism of $T$ with the given periodic data.

(3) $M=X_{n}, n \geqslant 2$. If $M$ is the connected sum of $n$ tori, then $\chi(M)=2-$ $2 n$ and by condition (b),

$$
\frac{\Pi_{i=1}^{l}\left(1-t^{q_{i}}\right) \Pi_{i=1}^{s}\left(1+t^{r_{i}}\right)}{(1-t)^{2 n-2} \Pi_{j=1}^{m}\left(1-t^{p_{j}}\right)}=1 .
$$

By Lemma 2.1, after reindexing the $q$ 's, we can write this product as

$$
\frac{(1-t)^{2 n-2}}{(1-t)^{2 n-2}} \prod_{i=1}^{m}\left[\frac{\left(1-t^{q_{i}}\right)\left(1+t^{q_{i}}\right)\left(1+t^{2 q_{i}}\right) \cdots\left(1+t^{2^{a_{i}-1} q_{i}}\right)}{\left(1-t^{2^{a_{i}} q_{i}}\right)}\right]=1 \text {. }
$$

Assume that $p_{1}=2^{a_{1}} q_{1}$ is the period of a source and $p_{m}=2^{a_{m}} q_{m}$ is the period of a sink. Let $T_{1}, \ldots, T_{n}$ be $n$ tori. By part (2), there exists a diffeomorphism $f_{1}$ of $T_{1}$ with periodic data

$$
\begin{aligned}
& \left\{(1,0,+1),(1,1,+1) ;\left(2^{a_{i}} q_{i}, u_{i},+1\right),\left(q_{i}, 1,+1\right),\right. \\
& \left.\quad\left(q_{i}, 1,-1\right), \ldots,\left(2^{a_{i}-1} q_{i}, 1,-1\right), i=1, \ldots, m-1\right\} .
\end{aligned}
$$

Again by (2), there exist diffeomorphisms $f_{2}, \ldots, f_{n-1}$ of $T_{2}, \ldots, T_{n-1}$ respectively such that $f_{i}$ has one sink of period 1 , one source of period 1 , and two saddles of period 1 and orientation type +1 .

Finally, let $f_{n}$ be a diffeomorphism of $T_{n}$ with periodic data

$$
\begin{array}{r}
\left\{(1,2,+1),(1,1,+1) ;\left(2^{a_{m}} q_{m}, 0,+1\right),\left(q_{m}, 1,+1\right),\right. \\
\left.\left(q_{m}, 1,-1\right), \ldots,\left(2^{a_{m}-1} q_{m}, 1,-1\right)\right\} .
\end{array}
$$

It is clear that the $f_{i}$ can be constructed so that there exists discs $D_{1} \subset$ $T_{1}, \ldots, D_{n-1} \subset T_{n-1}$ about the sinks of period 1 and discs $D_{2}^{\prime} \subset T_{2}, \ldots, D_{n}^{\prime}$ $\subset T_{n}$ about the sources of period 1 , and an $\varepsilon>0$, small compared to the diameter of the discs, such that $f_{i} \mid D_{i}$ is a contraction by $\varepsilon, i=1, \ldots, n-1$, and $f_{i} \mid D_{i}^{\prime}$ is an expansion by $\varepsilon, i=2, \ldots, n$. Let $R_{1} \subset D_{1}, \ldots, R_{n-1} \subset$ $D_{n-1}, R_{2}^{\prime} \subset D_{2}^{\prime}, \ldots, R_{n}^{\prime} \subset D_{n}^{\prime}$ be annuli of width $R$ containing the boundary of the disc, where $R$ is small compared to the diameters of the discs.

Now delete the discs $D_{i}-R_{i}$ and $D_{i}^{\prime}-R_{i}^{\prime}$ from $T_{i}$. For $i=1, \ldots, n-1$, 
identify the sets $R_{i}$ and $R_{i+1}^{\prime}$ by identifying $\partial R_{i} \cap \partial D_{i}$ with $\partial R_{i+1}^{\prime} \cap$ int $D_{i+1}^{\prime}$ and $\partial R_{i} \cap$ int $D_{i}$ with $\partial R_{i+1}^{\prime} \cap \partial D_{i+1}^{\prime}$. Then $X_{n}$ is the connected sum

$$
X_{n}=T_{1} \# T_{2} \# \cdots \# T_{n} \text {, }
$$

where $T_{i}$ is attached to $T_{i+1}$ by this identification. The identification is made so that $f_{i}$ and $f_{i+1}$ agree on $R_{i}$ (see Figure 10). Therefore, we can define a

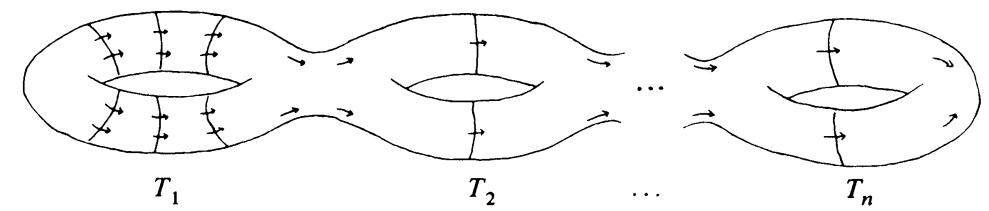

FIGURE 10

diffeomorphism $F$ of $X_{n}$ by setting $F(x)=f_{i}(x), x \in T_{i}$. Then $F$ has periodic data

$$
\begin{array}{r}
\left\{\left(2^{a_{i}} q_{i}, u_{i},+1\right),\left(q_{i}, 1,+1\right),\left(q_{i}, 1,-1\right), \ldots,\left(2^{a_{i}-1} q_{i}, 1,-1\right),\right. \\
i=1, \ldots, m ; \underbrace{(1,1,+1)}_{2 n-2 \text { times }}\} .
\end{array}
$$

If $N_{i}=T_{i} \# T_{i+1} \# \cdots \# T_{n}$, then

$$
X_{n}=N_{1} \supset N_{2} \supset \cdots \supset N_{n} \supset \varnothing
$$

satisfies the conditions of Lemma 3.4, so $F$ has a filtration on $X_{n}$. Therefore, by the $\Omega$-stability theorem and the Kupka-Smale theorem, there exists a Morse-Smale diffeomorphism $f$ of $X_{n}$ with the given periodic data.

(4) $M=P$. In this case, $\chi(M)=1$, and after reindexing the $q$ 's, the canonical form of the equation $(*)$ is

$$
\prod_{i=1}^{m}\left[\frac{\left(1-t^{q_{i}}\right)\left(1+t^{q_{i}}\right) \cdots\left(1+t^{2^{a_{i}-1} q_{i}}\right)}{\left(1-t^{2^{a_{i}} q_{i}}\right)}\right]=1, \quad \text { where } q_{1}=1 .
$$

Let $S^{2}=\left\{x \in \mathbf{R}^{3}|| x \mid=1\right\}$, and let $\sim$ be the relation on $S^{2}$ defined by identifying antipodal points of $S^{2}$ (so $x \sim y$ if and only if $y=x$ or $y=-x$ ). Then $P$ is the quotient $S^{2} / \sim$, and a diffeomorphism of $P$ is a diffeomorphism of $S^{2}$ which preserves the relation. We wish to construct a diffeomorphism of $P$ with periodic data

$$
\begin{aligned}
& \left\{\left(2^{a_{1}}, u_{1},+1\right),(1,1,-1), \ldots,\left(2^{a_{1}-1}, 1,-1\right) ;\left(2^{a_{i}} q_{i}, u_{i},+1\right),\right. \\
& \left.\quad\left(q_{i}, 1,+1\right),\left(q_{i}, 1,-1\right), \ldots,\left(2^{a_{i}-1} q_{i}, 1,-1\right), i=2, \ldots, m\right\},
\end{aligned}
$$

where $u_{i}=2$ for $k$ indices $i$ and $u_{i}=0$ for $m-k$ indices. 
Suppose $u_{1}=2$, so that $p_{1}=2^{a_{1}}$ is the period of a source. Then $u_{i}=0$ for some $i$, say $u_{m}$. Write $S^{2}$ as a union of subsets $S^{2}=A_{1} \cup \cdots \cup A_{2 m-1}$ with the following properties:

(a) $A_{1}$ is a disc with center $(0,0,1)$ and $A_{2}, \ldots, A_{m-1}$ are cylinders on the upper hemisphere of $S^{2}$ such that $A_{i} \cap A_{i+1}$ is a small annulus of width $R$, $i=1, \ldots, m-2$;

(b) $A_{m}$ is a cylinder containing the equator of $S^{2}$ (points $\left(x_{1}, x_{2}, x_{3}\right) \in S^{2}$ such that $x_{3}=0$ ) with the property that if $x \in A_{m}$, then $-x \in A_{m}$ and such that $A_{m} \cap A_{m-1}$ is an annulus of width $R$;

(c) for $i=m+1, \ldots, 2 m-1, A_{i}$ is the set of antipodal points of $A_{2 m-i}$.

Choose diffeomorphisms $f_{1}, \ldots, f_{m}$ defined on $A_{1}, \ldots, A_{m}$ respectively such that

(i) $f_{1}$ has periodic data

$$
\left\{\left(2^{a_{1}}, 2,+1\right),(1,1,-1),(2,1,-1), \ldots,\left(2^{a_{1}-1}, 1,-1\right)\right\}
$$

and $f_{1}$ maps $\partial A_{1}$ outside of $A_{1}$ (Lemma 3.1);

(ii) for $i=2, \ldots, m-1, f_{i}$ has periodic data

$$
\begin{array}{r}
\left\{\left(2^{a_{i}} q_{i}, u_{i},+1\right),\left(q_{i}, 1,+1\right),\left(q_{i}, 1,-1\right),\right. \\
\left.\left(2 q_{i}, 1,-1\right), \ldots,\left(2^{a_{i}-1} q_{i}, 1,-1\right)\right\}
\end{array}
$$

and $f_{i}$ maps $\partial A_{i} \cap A_{i-1}$ into $A_{i}$ and $\partial A_{i} \cap A_{i+1}$ out of $A_{i}$ (Lemma 3.3);

(iii) $f_{m}$ has periodic data

$$
\begin{array}{r}
\left\{\left(2^{a_{m}+1} q_{m}, 0,+1\right),\left(2 q_{m}, 1,+1\right),\left(2 q_{m}, 1,-1\right),\right. \\
\left.\left(2^{2} q_{m}, 1,-1\right), \ldots,\left(2^{a_{m}} q_{m}, 1,-1\right)\right\}
\end{array}
$$

and $f_{m}$ maps $\partial A_{m} \cap A_{m-1}$ and $\partial A_{m} \cap A_{m+1}$ into $A_{m}$ (Lemma 3.2). It is clear from the proof of Lemma 3.2 that $f_{m}$ can be constructed so that $f_{m}(x)=$ $-f_{m}(-x)$. In that case, $f_{m}^{n}(x)=-f_{m}^{n}(-x)$ for any $n$. Therefore, if $x_{i}$ is a periodic point of $f_{m}$ with period $k$, then

$$
f_{m}^{k}\left(-x_{i}\right)=-f_{m}^{k}\left(x_{i}\right)=-x_{i},
$$

so $-x_{i}$ is also periodic with period $k$.

For $i=m+1, \ldots, 2 m-1$, define $f_{i}$ with domain $A_{i}$ by $f_{i}(x)=$ $-f_{2 m-i}(-x)$. Then the periodic orbits of $f_{i}$ in $A_{i}$ are the antipodal points of the periodic orbits of $f_{2 m-i}$ in $A_{2 m-i}$.

Let $F(x)=f_{i}(x), x \in A_{i}$. Then $F$ is a diffeomorphism of $S^{2}$ such that $F(x)=-F(-x)$. Therefore, $F$ induces a diffeomorphism $\hat{F}$ of $P$. The periodic orbits in $A_{i}$ and $A_{2 m-i}$ are identified for $i=1, \ldots, m-1$, and in $A_{m} / \sim, \hat{F}$ has periodic data

$$
\left\{\left(2^{a_{m}} q_{m}, 0,+1\right),\left(q_{m}, 1,+1\right),\left(q_{m}, 1,-1\right), \ldots,\left(2^{a_{m}-1} q_{m}, 1,-1\right)\right\},
$$


since each orbit of $F \mid A_{m}$ consists of points $x$ and their antipodal points $-x$. Therefore, $\hat{F}$ has the given periodic data. Just as in (1), there is a subdivision of $P$ satisfying the conditions of Lemma 3.4. So there exists a filtration of $P$ for $\hat{F}$, and by the $\Omega$-stability theorem and the Kupka-Smale theorem, there exists a Morse-Smale diffeomorphism $f$ of $P$ with the given periodic data.

The case when $u_{1}=0$ follows in exactly the same way.

(5) $M=Y_{n}, n \geqslant 2$. If $M$ is the connected sum of $n$ projective planes, then $\chi(M)=2-n$. After reindexing the $q$ 's, the canonical form of the equation $(*)$ is

$$
\frac{(1-t)^{n-2}}{(1-t)^{n-2}} \prod_{i=1}^{m}\left[\frac{\left(1-t^{q_{i}}\right)\left(1+t^{q_{i}}\right)\left(1+t^{2 q_{i}}\right) \cdots\left(1+t^{2^{a_{i}-1} q_{i}}\right)}{\left(1-t^{2^{a_{i}}}\right)}\right]=1 .
$$

Let $P_{1}, \ldots, P_{n}$ be $n$ projective planes.

Assume that $p_{1}=2^{a_{1}} q_{1}$ is the period of a source and $p_{m}=2^{a_{m}} q_{m}$ is the period of a sink. By part (4), there exist diffeomorphisms $f_{1}, \ldots, f_{n}$ of $P_{1}, \ldots, P_{n}$ respectively with the following properties:

(a) $f_{1}$ has periodic data

$$
\begin{array}{r}
\left\{(1,0,+1) ;\left(2^{a_{i}} q_{i}, u_{i},+1\right),\left(q_{i}, 1,+1\right), \ldots,\right. \\
\left.\left(2^{a_{i}-1} q_{i}, 1,-1\right), i=1, \ldots, m-1\right\} ;
\end{array}
$$

(b) for $i=2, \ldots, n-1, f_{i}$ has one sink of period 1 , one source of period 1 , and one saddle of period 1 and orientation type +1 ;

(c) $f_{n}$ has periodic data

$$
\begin{aligned}
& \left\{(1,2,+1) ;\left(2^{a_{m}} q_{m}, 0,+1\right),\left(q_{m}, 1,+1\right),\right. \\
& \left.\left(q_{m}, 1,-1\right), \ldots,\left(2^{a_{m}-1} q_{m}, 1,-1\right)\right\} .
\end{aligned}
$$

Just as in part (3), the $f_{i}$ can be constructed so that if we delete discs about the sinks of period 1 in $P_{1}, \ldots, P_{n-1}$ and the sources of period 1 in $P_{2}, \ldots, P_{n}$ and attach the $P_{i}$ along the boundaries of these discs, then we can define a diffeomorphism $F$ of $Y_{n}=P_{1} \# P_{2} \# \cdots \# P_{n}$ by $F(x)=f_{i}(x), x \in$ $P_{i}$. Then $F$ has periodic data

$$
\begin{aligned}
&\left\{\left(2^{a_{i}} q_{i}, u_{i},+1\right),\left(q_{i}, 1,+1\right),\left(q_{i}, 1,-1\right), \ldots,\right. \\
&\left(2^{a_{i}-1} q_{i}, 1,-1\right), i=1, \ldots, m, \underbrace{(1,1,+1)}_{n-2 \text { times }}\} .
\end{aligned}
$$

Also, it is clear that $F$ has a filtration on $Y_{n}$. Therefore, by the $\Omega$-stability theorem and the Kupka-Smale theorem, there exists a diffeomorphism $f$ of $Y_{n}$ with the given periodic data. Q.E.D. 
4. Further consequences of the canonical form. The canonical form derived in Lemma 2.1 has several other consequences.

Proposition 4.1. Let $M$ be a compact, connected 2-manifold. Suppose $f$ is a Morse-Smale diffeomorphism of $M$ homotopic to the identity such that the orientation type of every saddle is -1 . Then

(a) $M=S^{2}$;

(b) all the periods of orbits of $f$ are powers of 2; in fact, there exist $n \geqslant 0$, $m \geqslant 0$ such that the periodic orbits of $f$ consist of one source of period $2^{n}$, one sink of period $2^{m}$, and $n+m$ saddles of periods $1,2, \ldots, 2^{n-1}, 1,2, \ldots, 2^{m-1}$ respectively.

Proof. Suppose $p_{1}, \ldots, p_{k}$ are the periods of sources and sinks of $f$ and $r_{1}, \ldots, r_{l}$ are the periods of saddles. Then

$$
\frac{(1-t)^{\chi(M)} \Pi_{i=1}^{l}\left(1+t^{r_{i}}\right)}{\Pi_{j=1}^{k}\left(1-t^{p_{j}}\right)}=1 .
$$

Since $f$ has at least one source and one sink, $k \geqslant 2$. Therefore, $\chi(M) \geqslant 2$, so $\chi(M)=2$. Hence, $M=S^{2}, k=2$, and by Lemma 2.1 , there exist $n \geqslant 0$, $m \geqslant 0$ such that $p_{1}=2^{n}, p_{2}=2^{m}$ and the set $\left\{r_{1}, \ldots, r_{l}\right\}$ equals the set $\left\{1,2, \ldots, 2^{n-1}, 1,2, \ldots, 2^{m-1}\right\}$. Q.E.D.

Note that the sphere is the only compact surface which admits a MorseSmale diffeomorphism with no saddles. In the case of other compact surfaces, a Morse-Smale diffeomorphism must have a certain minimal number of saddles. We state this as a lemma.

LEMMA 4.2. Let $M$ be a compact, connected 2-manifold and let $\lambda(M)$ be 0 , $2 n$, or $n$ depending on whether $M$ is a sphere, connected sum of $n$ tori $(n \geqslant 1)$, or connected sum of $n$ projective planes $(n \geqslant 1)$. Then a Morse-Smale diffeomorphism $f$ of $M$ which is homotopic to the identity must have at least $\lambda(M)$ saddles of orientation type +1 .

Proof. Suppose $f$ has $k$ sources and sinks and $l$ saddles of orientation type +1 . Then by Lemma $2.1, k=l+\chi(M)$. Since $k \geqslant 2, l \geqslant 2-\chi(M)$. Therefore, if $M=S^{2}, l \geqslant 0$; if $M$ is the connected sum of $n$ tori, $l \geqslant 2-(2-2 n)$ $=2 n$; and if $M$ is the connected sum of $n$ projective planes, $l \geqslant 2-(2-n)$ $=n$. Q.E.D.

We remark that it follows from the Morse-Smale inequalities [8] that any Morse-Smale diffeomorphism of $M$ must have at least $\lambda(M)$ saddles, although we can say nothing about the orientation type in this case.

If $f \in \operatorname{Diff}(M)$ has no more than $\lambda(M)$ saddles of orientation type +1 , then we have results similar to Proposition 4.1. 
Proposition 4.3. Let $M, \lambda(M)$ be as above, and let $f$ be a Morse-Smale diffeomorphism of $M$ which is homotopic to the identity. If $f$ has exactly $\lambda(M)$ saddles with orientation type +1 and period 1 , then there exist $n \geqslant 0, m \geqslant 0$ such that the other orbits of $f$ consist of one source of period $2^{n}$, one sink of period $2^{m}$ and $n+m$ saddles of periods $1,2, \ldots, 2^{n-1}, 1,2, \ldots, 2^{m-1}$.

The proof follows as in 4.1.

In general, given a fixed number of sources and sinks, we can construct a Morse-Smale diffeomorphism with an arbitrarily high number of saddles of orientation type -1 . The situation is different if we allow no such saddles.

Proposition 4.4. Let $f$ be a Morse-Smale diffeomorphism of a compact, connected 2-manifold homotopic to the identity, and suppose $f$ has no saddles of orientation type -1 . If $\chi(M) \geqslant 0$, then for any saddle of period $q$, $f$ has a corresponding sink or source of the same period. If $\chi(M)<0$, then $f$ has $-\chi(M)$ fixed point saddles and for any other saddle of period $q, f$ has $a$ corresponding sink or source of the same period.

Proof. Suppose $p_{1}, \ldots, p_{m}$ are the periods of sources and sinks of $f$, and $q_{1}, \ldots, q_{l}$ are the periods of saddles. Then

$$
\frac{(1-t)^{\chi(M)} \Pi_{i=1}^{l}\left(1-t^{q_{i}}\right)}{\Pi_{j=1}^{m}\left(1-t^{p_{j}}\right)}=1 .
$$

Suppose $\chi(M) \geqslant 0$. Then by Lemma 2.1 , the set $\left\{p_{1}, \ldots, p_{m}\right\}$ equals the set

$$
\{\underbrace{1, \ldots, 1}_{\chi(M) \text { times }}, q_{1}, \ldots, q_{l}\}
$$

and the result follows. If $\chi(M)<0$, then the set

$$
\{p_{1}, \ldots, p_{m}, \underbrace{1, \ldots, 1}_{-\chi(M) \text { times }}\}
$$

equals the set $\left\{q_{1}, \ldots, q_{l}\right\}$. Then we can reindex the $q$ 's so that $q_{1}=\cdots=$ $q_{-\chi(M)}=1$. Thus, there are $-\chi(M)$ fixed point saddles and for $i>\chi(M)$, there exists $j$ such that $p_{j}=q_{i}$. Q.E.D.

\section{BIBLIOGRAPHY}

1. R. Abraham and J. Robbin, Transversal mappings and flows, Benjamin, New York, 1967.

2. A. Dold, Fixed point index and fixed point theorem for Euclidean neighborhood retracts, Topology 4 (1965), 1-8.

3. J. Franks, Some smooth maps with infinitely many hyperbolic periodic points, Trans. Amer. Math. Soc. 226 (1977), 175-179. 1967.

4. W. Massey, Algebraic topology: an introduction, Harcourt, Brace and World, New York,

5. Z. Nitecki, Differentiable dynamics, M.I.T. Press, Cambridge, Mass., 1971. 
6. J. Palis and S. Smale, Structural stability theorems, Proc. Sympos. Pure Math., vol. 14, Amer. Math. Soc., Providence, R. I., 1970, pp. 223-231.

7. M. Peixoto, On an approximation theorem of Kupka and Smale, J. Differential Equations 3 (1967), 214-227.

8. S. Smale, Morse inequalities for a dynamical system, Bull. Amer. Math. Soc. 66 (1960), 43-49.

9. __ Differentiable dynamical systems, Bull. Amer. Math. Soc. 73 (1967), 747-817.

10. — The (-stability theorem, Proc. Sympos. Pure Math., vol. 14, Amer. Math. Soc., Providence, R. I., 1970, pp. 289-297.

Department of Mathematics, Northwestern University, Evanston, IllinoIs 60201 